\title{
The Theme of Risk Management
}

\author{
Chua David KH \\ Editor
}

Editorial

Available online June 11, 2014

The papers in this issue of the Journal come from different industry sectors, yet there can be a common theme that ties them together. Two of the papers address explicitly the issue of risk management, while the other three may be related to it in different degrees. One of the critical factors for project success is risk identification, as determined by Chua et al. (1999). The importance of risk management cannot be overemphasized. Failure to identify crucial risk elements in a project can lead to significant project failures in terms of cost and schedule.

The first paper examines the causes for disruptions, delays and failures in the delivery of IT-based services. The quality of service did not meet expectations when compared to that before the project. Among a number of key reasons for such failures, poor quality and risk management practices stand out with extremely low scoring, with the latter being the worst. A systematic framework comprising project management processes including a risk analysis plan was recommended.

The second paper looks at the persistent high rate of failure in Asia of projects of complex products and systems (CoPS). Specifically, it evaluates the maturity levels of risk management constructs in four perspectives: organizational context, risk management, project management and technological processes. With increased uncertainty, there is a corresponding increase in the maturity levels of some of these constructs, especially, of organizational culture, risk planning and identification, process improvement and integration and project management process to deal with the increased risk.

The subject of the third paper is on new product development. In particular, its focus is the risk management process of the food industry. Over the years, risk management systems have evolved from simple crisp "stage-gate" approach, or phase review process to rather sophisticated adaptable and overlapping stages with fuzzy conditional decision nodes. Through thorough literature review and extensive interviews with industry experts, a risk management process was proposed, dealing with 20 common risks identified in the industry.

The remaining papers deal with different subjects but yet have the notion of managing levels of risk in a project. One presents a mixed integer linear programming model to manage finite capacity scheduling of make-pack production. It incorporates rolling horizon scheduling to handle a dynamic environment to ensure robustness in the production schedule. The other looks at urban development to incorporate considerations for ethnic communities. The need here is to manage the multiple stakeholders' expectations and requirements to ensure project success.

At least in the construction industry, design can have a significant impact on cost risks. Figure 1 shows the typical effect of design effort on cost of late design changes. Building Information Modelling (BIM) (Van Nederveen and Tolman, 1992), offers a strategy to mitigate this risk through close collaboration and early design efforts. If design efforts can be moved upstream as shown in the arrow through sharing of information and incorporation of knowledge, the ability to control cost is tremendous (Chua, 2014). BIM offers the opportunity to virtually design and construct the building before large expense on time, resources and money (Khanzode et al., 2006). The digital model can be evaluated by various disciplines to ensure that the building performs to project specifications.

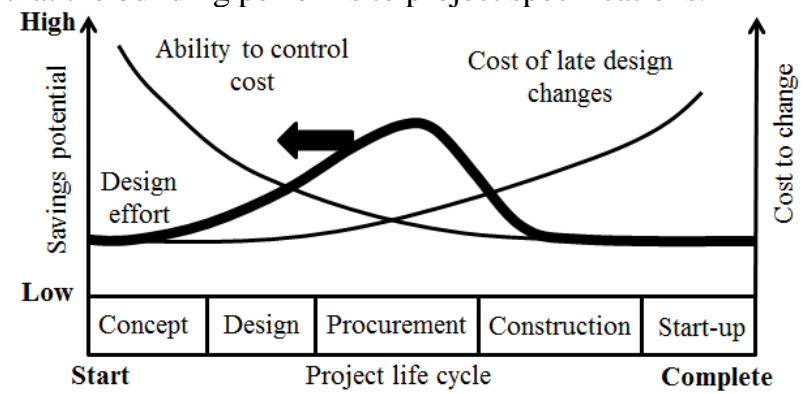

Fig. 1. Effort of design effort on cost to changes

In the context of the apparent theme of this issue, there remains the question of integrating the BIM workflow with risk management processes so that the potential of BIM can be fully exploited to mitigate risks downstream.

\section{References}

Chua, D. K. H., Kog, Y. C. and Loh, P. K. (1999). Critical success factors for different project objectives. Journal of construction engineering and management, 125 (3), 142-150.

Van Nederveen, G. A. and Tolman, F. P. (1992). Modelling multiple views on buildings. Automation in Construction, 1 (3), 215-24.

Chua, D. K. H. (2014). VDC: What it takes to go beyond BIM. Evening Talk Series, Institute of Engineers, Singapore.

Khanzode, A., Fischer, M., Reed, D. and Ballard, G. (2006). A Guide to Applying the Principles of Virtual Design \& Construction (VDC) to the Lean Project Delivery Process. CIFE Working Paper \#093, Stanford. 